\title{
Translation of genomics-guided RNA-based personalised cancer vaccines: towards the bedside
}

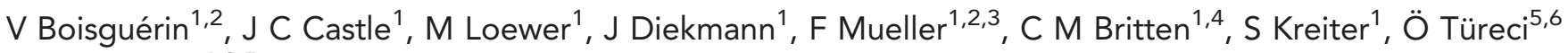 \\ and $U$ Sahin ${ }^{\star}, 1,2,5$ \\ ${ }^{1}$ TRON gGmbH-Translational Oncology at Johannes Gutenberg-University Medical Center gGmbH, Langenbeckstr 1, Building \\ 708, 55131 Mainz, Germany; ${ }^{2}$ BioNTech AG, Hölderlinstr 8, 55131 Mainz, Germany; ${ }^{3}$ TheraCode GmbH, Hölderlinstr 8, 55131 \\ Mainz, Germany; ${ }^{4}$ Ribological GmbH, Hölderlinstr 8, 55130 Mainz, Germany; ${ }^{5}$ University Medical Center of the Johannes \\ Gutenberg University Mainz, Mainz, Germany and ' Ganymed Pharmaceuticals AG, Freiligrathstraße 12, 55131 Mainz, Germany
}

Cancer is a disease caused by DNA mutations. Cancer therapies targeting defined functional mutations have shown clinical benefit. However, as $95 \%$ of the mutations in a tumour are unique to that single patient and only a small number of mutations are shared between patients, the addressed medical need is modest. A rapidly determined patient-specific tumour mutation pattern combined with a flexible mutation-targeting drug platform could generate a mutation-targeting individualised therapy, which would benefit each single patient. Next-generation sequencing enables the rapid identification of somatic mutations in individual tumours (the mutanome). Immunoinformatics enables predictions of mutation immunogenicity. Mutation-targeting RNA-based vaccines can be rapidly and affordably synthesised as custom GMP drug products. Integration of these cutting-edge technologies into a clinically applicable process holds the promise of a disruptive innovation benefiting cancer patients. Here, we describe our translation of the individualised RNA-based cancer vaccine concept into clinic trials.

Despite major advances in oncology, cancer still accounts for one in four of all deaths. As accumulation of genomic mutations constitutes a hallmark of cancer, the identification of causative 'driver' mutations shared by a subpopulation of patients and the subsequent design of small-molecule inhibitors against them is a classical blueprint in cancer drug development (Hait and Hambley, 2009). Drugs such as iamtininib (Glivec, Novartis, Basel, Switzerland) for treatment of BCR/ABL, vemurafenib (Zelboraf, Roche, Basel, Switzerland) for BRAF V600E and crizotinib (Xalkori, Pfizer, New York City, NY, USA) for EML4-ALK mutations provide unequivocal clinical benefit.

However, only a small number of mutations are shared between patients. Ninety-five per cent of the mutations in a patient's tumour appear to be unique to that tumour (Stratton, 2011). Moreover, only a fraction of the mutations are of biological relevance such that their functional inhibition is detrimental for the tumour cell and thus of therapeutic benefit. Conversely, simultaneous technological progress in two areas of highly synergistic potential, namely genomics and immunotherapy, has opened up conceptually novel paths to therapeutically exploit tumour mutations.

The advancement of sequencing technologies has revealed that human cancers carry dozens to hundreds of non-synonymous mutations (Shah et al, 2009). Genome-wide mutation identification initiatives have been launched, including the Cancer Genome Association and the International Cancer Genome Consortium. Indeed, with the emergence of next-generation sequencing (NGS), every patient's individual tumour mutanome 'signature' can be mapped within a short time frame and at a reasonable cost (Mardis and Wilson, 2009). This technological breakthrough in individualised tumour genomic diagnostics now calls for exploitation with individualised therapeutic approaches. Concepts are required to translate the comprehensive molecular cancer map of a patient to an on-demand, GMP, patient-specific therapeutic approach. 
Major advances in immunotherapy, specifically in the cancer vaccine field, enable us to exploit the individualised mutanome data. Only a small fraction of mutations may be critical for tumour survival and thus useful for function-inhibiting targeted approaches. The fraction of immunogenic mutations, in contrast, is considerably higher. Computational predictions suggest that tumours contain many antigenic mutations (Segal et al, 2008; Srivastava and Srivastava, 2009) and recent proof-of-concept studies in mice suggest that $25-30 \%$ of mutations found in tumours elicit T-cell immune responses (Castle et al, 2012). In contrast to the classical tumour-associated antigens that typically constitute autoantigens, mutations targetable by $\mathrm{T}$ cells are ideal vaccine targets, as they are not subject to central immune tolerance (Diekmann et al, 2012). The use of neo-epitopes as vaccine targets was demonstrated in a study showing nearly 50\% complete remissions in patients with vulvar intraepithelial neoplasia by vaccination with HPV16 E6 and E7 peptides (Kenter et al, 2009). Clinical studies have used mutated epitopes or even individually expressed mutations as vaccine targets (Abrams et al, 1996; Roth et al, 1996; Carbone et al, 2005; Rahma et al, 2010). Mutated tumour antigens tested have been assessed on the single-epitope level and induction of immune responses has been confirmed in patients (Lennerz et al, 2005) and as rejection antigens in mouse models (Matsushita et al, 2012). Emerging data support that poly-epitopic tumour-specific immune responses are predictive for therapeutic efficacy and are correlated with complete remissions in autologous patient-specific vaccines in advanced melanoma (Andersen et al, 2012). As tumours typically have tens to hundreds of non-synonymous mutations, the mutanome would provide many targets for poly-epitope vaccines for each individual patient.
Furthermore, novel drug platforms suitable for the on-demand production of GMP-quality individualised vaccines are emerging, including synthetic peptides (Yajima et al, 2005; Rammensee and Singh-Jasuja, 2013) and RNA (Kreiter et al, 2011b; Schlake et al, 2012). Among the different antigen formats for vaccination, coding messenger RNA (mRNA) is a particularly attractive option (Kreiter et al, 2010). Synthesised mRNA can be engineered to encode multiple types of transcripts, including synthetic poly-epitopic nucleotide sequences, and has a favourable safety profile (Kuhn et al, 2011). Further, RNA production is established, rapid and cost efficient.

Thus, a personalised therapy concept that integrates individualised tumour genome sequencing and on-demand RNA vaccine manufacture to create customised drug products is both an exciting new therapeutic vision and is within our grasp (Kreiter et al, 2011b) (Figure 1). We tested the analytical and therapeutic approach of such an individualised vaccine using the B16F10 murine melanoma model and the C57BL/6 background for (i) establishing an accurate method for identifying tumour mutations, (ii) testing the immunogenicity of a large list of confirmed tumour mutations and (iii) studying the efficacy of a cancer vaccine stimulating $\mathrm{T}$ cells specific for selected mutations. We have now moved this concept into clinic testing and, here, review our efforts and discuss the next steps.

\section{FROM THE BENCH: PRECLINICAL PROOF-OF-CONCEPT}

Using the B16F10 melanoma preclinical model, we conducted proof-of-concept experiments to demonstrate a process for somatic mutation identification, mutation selection, and on-demand

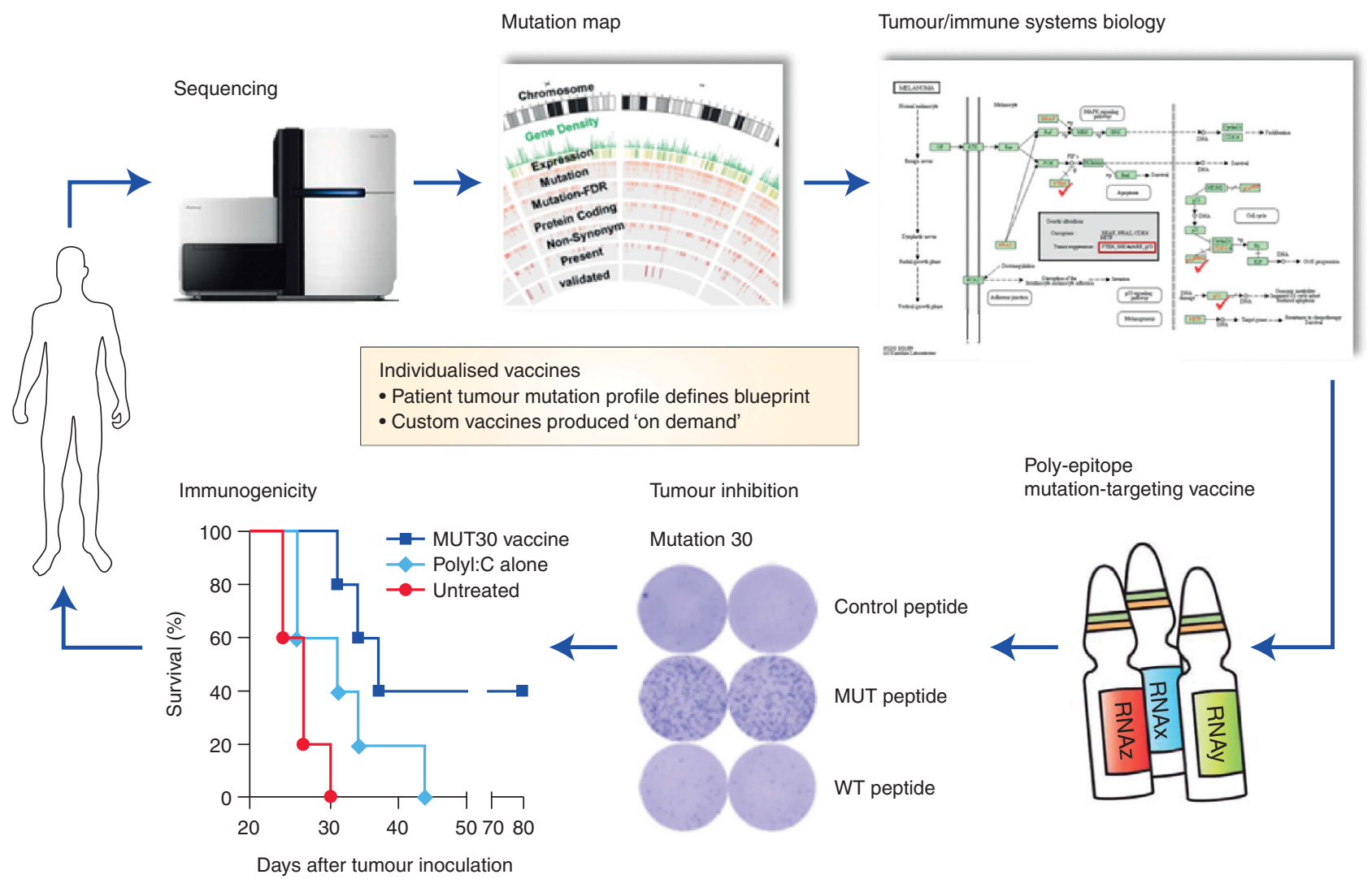

Figure 1. The individualised cancer vaccine concept. Mutations and gene expression are determined by NGS and bioinformatics. After identification of those mutations that have a likelihood of being immunogenic (T-cell-druggable mutanome), the blueprint for a unique, mutationtargeted vaccine is generated and the respective RNA-based vaccine is manufactured. In preclinical mouse proof-of-concept models, we have demonstrated that such vaccines activate mutation-specific T cells and mediate growth inhibition of established tumours. 
individualised vaccine production (Castle et al, 2012). B16F10 is a highly metastatic child clone of a cell line that spontaneously originated in a C57BL/6 mouse (Fidler, 1973). The B16F10 cell line, which is transplantable and expresses a variety of TAAs, is the most widely used model for experimental cancer therapies. In part, owing to low MHC expression (Boegel et al, 2013), B16 cells are poorly immunogenic, making B16F10 a model 'in which treatment is notoriously difficult' (Overwijk and Restifo, 2001). In sharp contrast to its frequent use as a preclinical model, there was little knowledge of the mutations underlying the malignant phenotype and thus the repertoire of potential immunotherapy targets.

To identify the cancer mutanome, consisting of tumour-specific protein-coding mutations, we sequenced the protein-coding genome, the exome, of B16F10 cells and of C57BL/6 wild-type mouse cells. In this first sequencing of a mouse tumour worldwide, more than 31 billion nucleotides were generated. We captured and sequenced each exome in triplicate. We also sequenced the B16F10 transcriptome using RNA-Seq to determine tumour gene expression.

We used existing algorithms for NGS data processing ( $\mathrm{Li}$ and Durbin, 2009), mutation discovery (Li et al, 2009; Castle et al, 2010; Larson et al, 2012), and gene expression (Langmead et al, 2009). However, existing tools to identify tumour-specific single-nucleotide point variations have poor congruence, which is not acceptable if data for a diagnostic procedure define a therapeutic intervention. Therefore, we developed a novel statistical framework incorporating replicate exome sequencing to identify and prioritise true somatic mutations (Lower et al, 2012). Mutations were discovered in crucial signalling pathways (e.g., RAS/MAPK/ERK and PIEK/AKT), in the DNA repair machinery and in genes of relevance to oncogenesis (e.g., Alk, Flt1 and Fat1). This provided a comprehensive potential set of target mutations, but the challenge of selecting a set of candidates for an individual poly-epitopic vaccine remained.

We designed a rational approach to select mutations qualifying for immunogenicity testing based on the following criteria: (i) present in all B16F10 triplicates and absent in all C57BL/6 triplicates, (ii) high statistical confidence as a true mutation, (iii) in a protein-coding gene that is expressed in $\mathrm{B} 16 \mathrm{~F} 10$, (iv) causes a non-synonymous protein change $(\mathrm{v})$ and predicted to be in a peptide presented on B16F10 MHC molecules (Lower et al, 2012; Lundegaard et al, 2008). We identified 3570 somatic mutations, including 1392 in transcripts, of which 1266 were in proteincoding regions (see Table 1). Of the 1266 mutations in proteincoding regions, 962 cause non-synonymous protein changes and, of these, 563 occur in an expressed gene based on the RNA-Seq sequencing. Of the 563 expressed non-synonymous mutations, we selected 50 and confirmed all 50 by PCR and Sanger sequencing.

For testing their capability to induce mutation-specific T-cell responses, naive $\mathrm{C} 57 \mathrm{BL} / 6$ mice were vaccinated with long synthetic peptides corresponding to the mutant proteins with poly(I:C) as adjuvant (Kreiter et al, 2010). The response of splenocytes to RNAs encoding the mutated antigen and transfected into dendritic cells was evaluated by ELISPOT assays (Kreiter et al, 2007). One-third of the mutated epitopes were strongly immunogenic and as efficient as the positive control Trp2, the strongest known B16F10 murine melanoma antigen. In the majority of cases, the induced $\mathrm{T}$-cell response preferentially recognised the mutated epitopes rather than the respective wild types. No correlation between the immunogenicity and the potential oncogenicity of the target molecule ('driver' vs 'bystander' mutations) was observed.

In a syngeneic mouse system, we assessed whether immune responses elicited by immunisation with mutations translate into anti-tumoural effects. Prophylactic immunisation with $27 \mathrm{mer}$ peptides encoding these mutations achieved complete tumour protection and survival in $40 \%$ of the mice, whereas all mice in the control group died within 44 days. In the immunised mice that developed tumours, tumour growth was delayed and median

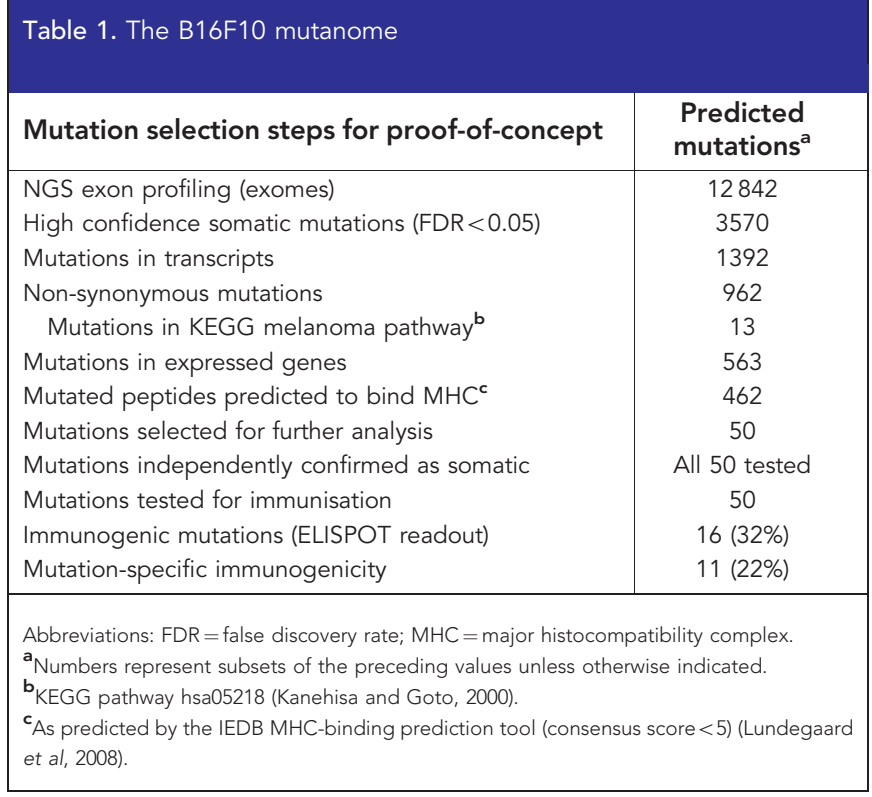

survival increased. In the therapeutic setting, the immunisation significantly delayed tumour growth.

\section{TOWARDS THE BEDSIDE: PREPARING FOR CLINICAL} TRIALS

These studies represent a preclinical proof-of-concept that demonstrate that, in advanced mouse models, a mutanome-targeting vaccine can elicit potent immunogenicity and confer tumour growth inhibition and control.

For clinical implementation of this approach, several challenges remain to be solved. The entire process, from patient sample through individualised drug product back to the patient, has to be rapid, has to be robust and must comply with the regulatory requirements of a controlled drug development process defined by drug development guidelines. Among the key challenges is the establishment of processes leading to the set of mutations to be used for the individual patient, the on-demand manufacturing of the poly-epitopic mutation-based vaccine and the appropriate clinical trial concepts and regulatory approval.

\section{FRONT-END OF THE PROCESS: GENERATING THE} MUTANOME MAP FROM A CLINICAL SAMPLE

NGS-based diagnostics is a state-of-the art platform allowing unbiased interrogation of nucleic acids; however, its current use is primarily in research and development settings. Starting with sample logistics to the reporting of validated and confirmed mutations for a given patient sample, the platform has to be adapted for clinical use. Genomic analysis of clinical samples requires an ethics approval and patient-informed consent explicitly allowing analysis of patient genomes. Auditable standard operating procedures have to be in place for the acquisition, handling, transport and documentation of patient sample material. Once in the biobank, the reception, labelling, processing, storing and tracking of each sample and its derivatives must be done correctly and correctly documented. This process should be coupled to an efficient laboratory information management system for the collection and documentation of all operational steps and the resultant data (Scholtalbers et al, 2013). Relevant clinical 
annotation and outcome information must be securely and privately stored.

For a clinical-grade NGS process, clear input and output criteria, controlled reagents and calibration processes must be defined for each step. The nucleic acid extraction protocols will vary depending on the sample and must be optimised for each type of input material, such as fresh frozen tissue and blood, and derivatives (RNA and DNA). Extraction of RNA and DNA and NGS library preparation from formalin-fixed and paraffinembedded tissues is challenging but required as these are the most commonly used methods of tissue preservation. Exome resequencing kits are available from different vendors: the protocols, the library preparation steps, the target regions and the data generated differ for each kit. Gene expression profiling by NGS RNA-Seq is a robust and established process, but variations in vendor kits change measured gene expression. Thus, for the use of vendor-supplied kits, clear standard operating procedures, effective calibration for each sample type, defined workflows, and reagent and lot number tracking are required.

Best practices for the computational analyses of NGS data are rapidly evolving. Nevertheless, for any clinical application, the workflows need to be defined in standard operating procedures, ensuring data reproducibility, accessibility and privacy. Moreover, key software components need to be verified and validated as well as optimised for speed of execution. Software used during the trial must be in repositories and 'locked down' to prevent unanticipated changes.

We demonstrated that replicate DNA sequencing reduces the number of false-positive mutation calls, as measured by Sanger sequencing (Lower et al, 2012), and are thus using replicate DNA tumour and germline sequencing followed by independent mutation confirmation. Genomic positions with predicted mutations can be amplified with patient-specific PCR assays for Sanger sequencing of the tumour and PBMC (germline) samples. As DNA upstream and downstream of the predicted mutation is also determined by the Sanger sequencing, the resultant sequence also allows for verification of assay specificity. These steps confirm that a mutation exists in the patient tumour genome and that it does not exist in the patient germline genome.

We anticipate multiple improvements in the mutation detection workflow. First, the use of replicate sequencing increases costs and necessitates additional input nucleic acid amounts. Second, while robotics and lab automation simplify the pipetting of the tens to hundreds of PCRs primers for the Sanger sequencing, the use of Sanger sequencing would be prohibitive for a phase III study. Further, Sanger sequencing of mutations has a limited detection level, particularly for heterozygous mutations in impure, heterogeneous tumour samples. Optimised bioinformatics algorithms, enhanced library construction methods and third-generation sequencing should increase the accuracy, robustness and reproducibility of NGS-based mutation detection while decreasing the costs and time. We expect the incorporation of these expected improvements and their acceptance by regulatory authorities should thus eliminate the need for replicates and independent Sanger sequencing for mutation confirmation.

\section{READY-TO-GO: CREATING THE PATIENT'S PERSONAL} VACCINE DRUG

On the basis of the confirmed mutanome map of a patient's tumour, mutations are selected for the vaccines that have a higher likelihood of conveying tumour control. A mutation to be incorporated into a poly-epitopic vaccine should (i) be confirmed by independent methods, (ii) be specific to the tumour sample and not in the patient germline genome, (iii) occur in a protein-coding transcript, (iv) cause a change in protein sequence and (v) be expressed in the tumour cells. Tumour gene expression can be determined using RNA-Seq to profile the patient's tumour. A challenging aspect is the selection of mutations that are antigenic and capable of inducing a robust $\mathrm{T}$-cell response recognising the respective mutated epitope. This requires that the mutationcontaining epitope is presented on the patient's MHC molecules, in both the dendritic (during the vaccination) and tumour cells (for tumour recognition). With the patient HLA haplotype, MHCbinding epitopes can be predicted with algorithms such as those from the immune epitope database (Lundegaard et al, 2008). We have developed a bioinformatic approach for determination of HLA haplotypes directly from RNA-Seq data, called seq2HLA (Boegel et al, 2013). Seq2HLA additionally provides information about expression levels of the respective HLA-alleles in the tumour sample, which may further facilitate the mutation selection process. Furthermore, it is possible to test the immunogenicity of the computationally predicted epitopes using short-time in vitro T-cell stimulation approaches (Kreiter et al, 2007). The use of the patient's own lymphocytes in such assays allows identification of mutations that have already induced a spontaneous T-cell response in the tumour-bearing patient.

Once the patient's individualised vaccine has been designed, the vaccine blueprint must be manufactured in a custom, GMPcompliant, small-scale batch specific for the single patient. The manufacturing process and individual batch production costs are thus particular challenges. Recombinant antigen-encoding RNA is an attractive technology platform for rapid GMP-compliant manufacturing of vaccines that are tailored to the mutation signature of an individual patient's tumour (Kreiter et al, 2011b). RNA is easy and fast to synthesise in a GMP-compliant manner and RNA does not integrate into the host genome, facilitating regulatory hurdles. RNA vaccines have a number of additional advantages that make them potent, safe and versatile. They (i) have an intrinsic adjuvant activity, (ii) can represent multiple epitopes in a single molecule, (iii) are endogenously translated and effectively enter the endogenous antigen processing and presentation machinery, and (iv) elicit potent anti-tumoural immunity (Kreiter et al, 2010; Diken et al, 2011; Kuhn et al, 2011; Kreiter et al, 2011a, b; Diken et al, 2013). One of the key challenges is the in vivo delivery of an mRNA vaccine, as mRNA does not easily transit through the cell membrane and is degraded by ubiquitous extracellular RNases. There are different ways to address delivery. Vaccine administration can be done using RNA-transfected dendritic cells and direct mRNA administration. Relative to autologous DCs, RNA has the advantage of lower costs, use in outpatient clinics, and less complicated logistics, thus making RNA vaccines available 'off-the-shelf' to patients. Both intranodal as well as the intradermal injections of RNA are feasible, have demonstrated delivery to DCs and efficacious inducement of $\mathrm{T}$ cells (Diken et al, 2011) and are currently under clinical investigation (Weide et al, 2008; Kreiter et al, 2010). Finally, liposomal RNA vaccination approaches are under development, which would allow a simpler intravenous application for systemic RNA vaccination.

BACK-END OF THE PROCESS: REACHING CLINICAL STAGE AND THE MARKET

The individualised vaccine concept must be tested in clinical trials: the entire process from sample acquisition, mutation discovery, vaccine design and production, drug administration and clinical monitoring must be run in the framework of a regulatoryapproved clinical trial. Regulatory risks for the clinical development of individualised mutanome vaccines arise owing to the fact that despite highly standardised processes for the mutation identification and selection, the vaccine design and the 
manufacturing of the drug products, each patient will receive a tailored vaccine that will be unique and therefore vary between patients. This is fundamentally different to classical molecularly defined vaccines that can be pre-manufactured, toxicity tested and warehoused before use. This paradigm shift creates a series of challenges for drug developers, drug suppliers, physicians and regulators that have been intensively discussed over a period of several years. On the basis of these discussions, the CIMT regulatory research group proposed regulatory principles for clinical development of individualised vaccines. Following a meeting with the Innovation Task Force at the European Medicine Agency, the regulatory agency endorsed the proposed blueprint for clinical development of such innovative new therapies. It was clarified that the development of individualised vaccines differs from the development of existing cancer therapeutics and that the existing EU regulatory framework does not address individualised vaccines. Thus, it was identified both that some flexibility in the interpretation of the existing guidance would be needed and that several regulatory principles already in place for the development of cellular and autologous therapeutics may be applicable to the development and testing of recombinant individualised vaccines (Britten et al, 2013). Thus, many regulatory challenges have been identified and are being actively addressed in conjunction with the appropriate regulatory authorities, resulting in blueprints that are already published.

Safety is one key aspect requiring particular attention in the clinical translation of novel therapeutic drugs. As the sequence composition of the individualised drug product will be patientspecific, formal preclinical toxicity studies are not feasible. Thus, preclinical toxicity studies must be designed in accordance with FDA (US Food and Drug Administration), International Conference on Harmonisation and Committee for Medicinal Products for Human Use guidelines to test the safety of the approach based on a representative process and drug products.

A vaccine encoding a mutation-containing peptide might induce a T-cell reaction against the wild-type analogue peptide that is expressed by healthy tissues. Our experience in preclinical models is that vaccination with mutated epitopes can result in the induction of $\mathrm{T}$ cells that recognise non-mutated epitopes. Even though cancer vaccines have been demonstrated to be very safe and we have not seen evidence of autoimmunity-associated toxicities in mouse models, toxicity by recognition of the wild-type epitope in normal tissues cannot be fully excluded. Further, the target antigens used for the individualised vaccine will differ for every patient, resulting in a variation of potential adverse effects mediated by this toxicity. Therefore, diligent safety monitoring of patients is mandatory and the investigators and clinicians should be provided with information listing in which organs each wildtype gene is highly expressed.

The pharmacodynamic activity of individualised mutanome vaccines will need to be assessed by clinical, molecular and immunological endpoint assessments throughout the clinical development. The classical clinical endpoints such as survival and progression free survival will have a major role in the later stages of clinical development aiming for proof of efficacy and marketing authorisation. Imaging of tumour lesions should be implemented early on in clinical development to capture first hints of potential efficacy in patients with measurable disease. Quantification of potential surrogates of tumour cell burden in the body, such as circulating tumour cells and circulating nucleic acids and proteins, may allow identification of alternative signatures guiding the further clinical development of individualised vaccines. Quantifying the frequency, function and phenotype of immune cell subsets following therapy in the peripheral blood, in the skin following the induction of delayed type hypersensitivity reactions, and most importantly in the tumour may identify additional biomarkers. Following the anticipated mode of action of the individualised vaccines, the induction of functional mutationspecific T-cell responses, as well as their trafficking to the tumour as determined by functional immunological readouts, and TCR repertoire profiling are straightforward strategies to achieve the clinical proof-of-concept first in human trials. Longitudinally measuring antigen-specific T cells in blood samples and in tumour biopsies at multiple time points before and after vaccination will be instructive to understand the immunogenicity of each individual mutation. Immunogenicity data generated from such studies will be used to further optimise and refine the target selection for the individualised vaccination approach. Combining the individualised mutanome-vaccine approach with potent immunomodulatory therapies, such as anti-checkpoint inhibitor antibodies, will open additional avenues for further clinical development (Garber, 2013). Whereas the immunomodulatory therapy would release the brakes of the immune system, the individualised vaccine approach would instruct the immune system by providing a tailored target map to maximise anti-tumoural effects.

In addition to the regulatory and clinical challenges, the pharmacoeconomy of individualised treatments poses a development risk owing to restricted budgets in health-care systems. In their early phase of clinical development, individualised vaccines will be expensive, mainly owing to costs for genome sequencing and manufacture of small, patient-specific GMP drug product batches. Commercialisation of individualised vaccines to reach larger number of patients will require not only evidence for high 'game-changing' clinical efficacy but also reduction of costs for providing a personalised treatment. There is good reason to believe that cost-effective delivery of vaccines can be achieved in the future, not only owing to decreasing genome sequencing costs but also owing to full automation and optimisation of the manufacturing process and the economy of scales that will reduce costs per batch for starting materials, release testing, and the overhead associated with the manufacturing facility. Indeed, regulatory, clinical and pharmacoeconomic challenges have been identified and are already being addressed.

\section{CONCLUSION}

Genomic-engineered individualised therapies may have substantial benefits as human cancer therapies. Rather than shy away from patient tumour heterogeneity, this concept exploits the unique mutations present in each individual tumour for the benefit of the patient. The individualised vaccines target the rich T-celldruggable mutanome, exploiting the mutations by combining NGS discovery with systematic immunogenicity analysis of mutations, followed by on-demand RNA drug synthesis. In advanced disease with unstable tumour genomes, tumours evolve in response to targeted therapies: individualised T-cell therapies may perform better than other treatment options, as the accumulation of mutations results in more antigens targetable by this flexible platform. Markedly reducing the costs and time required for genome-wide discovery of cancer-specific mutations, such as with 'third-generation' instruments, will further open the door for individualised immunotherapy of cancer patients. As for any new therapeutic approach, close collaboration with regulatory agencies is required to define the framework that enables the safe development and testing of this new, exciting and promising therapeutic concept.

We have received regulatory approval and begun enrolment in RNA-based individualised vaccines for cancer) for melanoma. Worldwide, similar mutation-targeting vaccination efforts have been initiated for glioblastoma (GAPVAC, http://www.gapvac.eu/), paediatric acute lymphoblastic leukaemia (iVacALL), hepatocellular carcinoma (HEPAVAC, http://www.hepavac.eu/), and triple 
negative breast tumour (MERIT, http://merit-consortium.eu/) patients. This approach opens a new dimension for individualised immunotherapy, adding tailored vaccine concepts as anti-tumour therapeutics.

\section{ACKNOWLEDGEMENTS}

Research described here was funded in part by BMBF grants 01GU1103A, 131A004A, 0316179A, the BMBF Go-Bio initiative and the state of Rhineland-Palatinate.

\section{REFERENCES}

Abrams SI, Hand PH, Tsang KY, Schlom J (1996) Mutant ras epitopes as targets for cancer vaccines. Semin Oncol 23(1): 118-134.

Andersen RS, Thrue CA, Junker N, Lyngaa R, Donia M, Ellebaek E, Svane IM, Schumacher TN, Thor Straten P, Hadrup SR (2012) Dissection of T-cell antigen specificity in human melanoma. Cancer Res 72(7): $1642-1650$.

Boegel S, Lower M, Schafer M, Bukur T, de Graaf J, Boisguerin V, Tureci O, Diken M, Castle JC, Sahin U (2013) HLA typing from RNA-Seq sequence reads. Genome Med 4(12): 102.

Britten CM, Singh-Jasuja H, Flamion B, Hoos A, Huber C, Kallen KJ, Khleif SN, Kreiter S, Nielsen M, Rammensee HG, Sahin U, Hinz T, Kalinke U (2013) The regulatory landscape for actively personalized cancer immunotherapies. Nat Biotechnol 31(10): 880-882.

Carbone DP, Ciernik IF, Kelley MJ, Smith MC, Nadaf S, Kavanaugh D, Maher VE, Stipanov M, Contois D, Johnson BE, Pendleton CD, Seifert B, Carter C, Read EJ, Greenblatt J, Top LE, Kelsey MI, Minna JD, Berzofsky JA (2005) Immunization with mutant p53- and K-ras-derived peptides in cancer patients: immune response and clinical outcome. J Clin Oncol 23(22): 5099-5107.

Castle JC, Biery M, Bouzek H, Xie T, Chen R, Misura K, Jackson S, Armour CD, Johnson JM, Rohl CA, Raymond CK (2010) DNA copy number, including telomeres and mitochondria, assayed using next-generation sequencing. BMC Genomics 11(1): 244.

Castle JC, Kreiter S, Diekmann J, Lower M, van de Roemer N, de Graaf J, Selmi A, Diken M, Boegel S, Paret C, Koslowski M, Kuhn AN, Britten CM, Huber C, Tureci O, Sahin U (2012) Exploiting the mutanome for tumor vaccination. Cancer Res 72(5): 1081-1091.

Diekmann J, Loewer M, Castle JC, Kreiter S, Tuereci O, Sahin U (2012) The T cell druggable genome. Eur Pharm Rev 17(4): 16-19.

Diken M, Kreiter S, Selmi A, Britten CM, Huber C, Tureci O, Sahin U (2011) Selective uptake of naked vaccine RNA by dendritic cells is driven by macropinocytosis and abrogated upon DC maturation. Gene Ther 18(7): 702-708.

Diken M, Kreiter S, Selmi A, Tureci O, Sahin U (2013) Antitumor vaccination with synthetic mRNA: strategies for in vitro and in vivo preclinical studies. Methods Mol Biol 969: 235-246.

Fidler IJ (1973) Selection of successive tumour lines for metastasis. Nat New Biol 242(118): 148-149.

Garber K (2013) Melanoma combination therapies ward off tumor resistance. Nat Biotechnol 31(8): 666-668.

Hait WN, Hambley TW (2009) Targeted cancer therapeutics. Cancer Res 69(4): 1263-1267, discussion 1267.

Kanehisa M, Goto S (2000) KEGG: kyoto encyclopedia of genes and genomes. Nucleic Acids Res 28(1): 27-30.

Kenter GG, Welters MJ, Valentijn AR, Lowik MJ, Berends-van der Meer DM, Vloon AP, Essahsah F, Fathers LM, Offringa R, Drijfhout JW, Wafelman AR, Oostendorp J, Fleuren GJ, van der Burg SH, Melief CJ (2009) Vaccination against HPV-16 oncoproteins for vulvar intraepithelial neoplasia. $N$ Engl $J$ Med 361(19): 1838-1847.

Kreiter S, Diken M, Selmi A, Diekmann J, Attig S, Husemann Y, Koslowski M, Huber C, Tureci O, Sahin U (2011a) FLT3 ligand enhances the cancer therapeutic potency of naked rna vaccines. Cancer Res 71: 6132-6142.

Kreiter S, Diken M, Selmi A, Tureci O, Sahin U (2011b) Tumor vaccination using messenger RNA: prospects of a future therapy. Curr Opin Immunol 23(3): 399-406.
Kreiter S, Konrad T, Sester M, Huber C, Tureci O, Sahin U (2007) Simultaneous ex vivo quantification of antigen-specific CD4 + and $\mathrm{CD} 8+\mathrm{T}$ cell responses using in vitro transcribed RNA. Cancer Immunol Immunother 56(10): 1577-1587.

Kreiter S, Selmi A, Diken M, Koslowski M, Britten CM, Huber C, Tureci O, Sahin U (2010) Intranodal vaccination with naked antigen-encoding RNA elicits potent prophylactic and therapeutic antitumoral immunity. Cancer Res 70(22): 9031-9040.

Kuhn AN, Diken M, Kreiter S, Vallazza B, Tureci O, Sahin U (2011) Determinants of intracellular RNA pharmacokinetics: Implications for RNA-based immunotherapeutics. RNA Biol 8(1): 35-43.

Langmead B, Trapnell C, Pop M, Salzberg SL (2009) Ultrafast and memory-efficient alignment of short DNA sequences to the human genome. Genome Biol 10(3): R25.

Larson DE, Harris CC, Chen K, Koboldt DC, Abbott TE, Dooling DJ, Ley TJ, Mardis ER, Wilson RK, Ding L (2012) SomaticSniper: identification of somatic point mutations in whole genome sequencing data. Bioinformatics 28(3): 311-317.

Lennerz V, Fatho M, Gentilini C, Frye RA, Lifke A, Ferel D, Wolfel C, Huber C, Wolfel T (2005) The response of autologous T cells to a human melanoma is dominated by mutated neoantigens. Proc Natl Acad Sci USA 102(44): 16013-16018.

Li H, Durbin R (2009) Fast and accurate short read alignment with burrows-wheeler transform. Bioinformatics 25(14): 1754-1760.

Li H, Handsaker B, Wysoker A, Fennell T, Ruan J, Homer N, Marth G, Abecasis G, Durbin R (2009) The sequence alignment/map format and SAMtools. Bioinformatics 25(16): 2078-2079.

Löwer M, Renard BY, de Graaf J, Wagner M, Paret C, Kneip C, Tureci O, Diken M, Britten C, Kreiter S, Koslowski M, Castle JC, Sahin U (2012) Confidence-based somatic mutation evaluation and prioritization. PLoS Comput Biol 8(9): e1002714.

Lundegaard C, Lamberth K, Harndahl M, Buus S, Lund O, Nielsen M (2008) NetMHC-3.0: accurate web accessible predictions of human, mouse and monkey MHC class I affinities for peptides of length 8-11. Nucleic Acids Res W509-W512.

Mardis ER, Wilson RK (2009) Cancer genome sequencing: a review. Hum Mol Genet 18(R2): R163-R168.

Matsushita H, Vesely MD, Koboldt DC, Rickert CG, Uppaluri R, Magrini VJ, Arthur CD, White JM, Chen YS, Shea LK, Hundal J, Wendl MC, Demeter R, Wylie T, Allison JP, Smyth MJ, Old LJ, Mardis ER, Schreiber RD (2012) Cancer exome analysis reveals a T-cell-dependent mechanism of cancer immunoediting. Nature 482(7385): 400-404.

Overwijk WW, Restifo NP (2001) B16 as a mouse model for human melanoma. Curr Protoc Immunol Chapter 20: Unit 201.

Rahma OE, Ashtar E, Ibrahim R, Toubaji A, Gause B, Herrin VE, Linehan WM, Steinberg SM, Grollman F, Grimes G, Bernstein SA, Berzofsky JA, Khleif SN (2010) A pilot clinical trial testing mutant von Hippel-Lindau peptide as a novel immune therapy in metastatic renal cell carcinoma. J Transl Med 8: 8.

Rammensee HG, Singh-Jasuja H (2013) HLA ligandome tumor antigen discovery for personalized vaccine approach. Expert Rev Vaccines 12(10): 1211-1217.

Roth J, Dittmer D, Rea D, Tartaglia J, Paoletti E, Levine AJ (1996) p53 as a target for cancer vaccines: recombinant canarypox virus vectors expressing p53 protect mice against lethal tumor cell challenge. Proc Natl Acad Sci USA 93(10): 4781-4786.

Schlake T, Thess A, Fotin-Mleczek M, Kallen KJ (2012) Developing mRNA-vaccine technologies. RNA Biol 9(11): 1319-1330.

Scholtalbers J, Rossler J, Sorn P, de Graaf J, Boisguerin V, Castle J, Sahin U (2013) Galaxy LIMS for next-generation sequencing. Bioinformatics 29: 1233-1234.

Segal NH, Parsons DW, Peggs KS, Velculescu V, Kinzler KW, Vogelstein B, Allison JP (2008) Epitope landscape in breast and colorectal cancer. Cancer Res 68(3): 889-892.

Shah SP, Morin RD, Khattra J, Prentice L, Pugh T, Burleigh A, Delaney A, Gelmon K, Guliany R, Senz J, Steidl C, Holt RA, Jones S, Sun M, Leung G, Moore R, Severson T, Taylor GA, Teschendorff AE, Tse K, Turashvili G, Varhol R, Warren RL, Watson P, Zhao Y, Caldas C, Huntsman D, Hirst M, Marra MA, Aparicio S (2009) Mutational evolution in a lobular breast tumour profiled at single nucleotide resolution. Nature 461(7265): 809-813.

Srivastava N, Srivastava PK (2009) Modeling the repertoire of true tumor-specific MHC I epitopes in a human tumor. PLoS One 4(7): e6094. 
Stratton MR (2011) Exploring the genomes of cancer cells: progress and promise. Science 331(6024): 1553-1558.

Weide B, Garbe C, Rammensee HG, Pascolo S (2008) Plasmid DNA- and messenger RNA-based anti-cancer vaccination. Immunol Lett 115(1): 33-42.

Yajima N, Yamanaka R, Mine T, Tsuchiya N, Homma J, Sano M, Kuramoto T, Obata Y, Komatsu N, Arima Y, Yamada A, Shigemori M, Itoh K, Tanaka R (2005) Immunologic evaluation of personalized peptide vaccination for patients with advanced malignant glioma. Clin Cancer Res 11(16): 5900-5911.

(c) (1) (2) (2) This work is licensed under the Creative Commons (c) ${ }_{\text {BY }}$ SA Attribution-NonCommercial-Share Alike 3.0 Unported License. To view a copy of this license, visit http://creativecommons. org/licenses/by-nc-sa/3.0/ 